\title{
AN INNOVATIVE PROTOCOL FOR COMPARING PROTEIN BINDING SITES VIA ATOMIC GRID MAPS
}

\author{
M. Bicego ${ }^{1,2}$, A. D. Favia ${ }^{3}$, P. Bisignano ${ }^{3}$, A. Cavalli ${ }^{3}$, V. Murino ${ }^{1,2}$ \\ ${ }^{1}$ PLUS Lab, Istituto Italiano di Tecnologia, I-16163 Genoa, Italy \\ ${ }^{2}$ Dipartimento di Informatica (University of Verona), I-37134 Verona, Italy \\ ${ }^{3}$ Department of Drug Discovery and Development (D3), Istituto Italiano di Tecnologia, I-16163 Genoa, Italy
}

Keywords: Protein similarity, 3D points alignment, Iterative Closest Point, Drug design, Multitarget

\begin{abstract}
This paper deals with a novel computational approach that aims to measure the similarities of protein binding sites through comparison of atomic grid maps. The assessment of structural similarity between proteins is a longstanding goal in biology and in structure-based drug design. Instead of focusing on standard structural alignment techniques, mostly based on superposition of common structural elements, the proposed approach starts from a physicochemical description of the proteins' binding site. We call these atomic grid maps. These maps are preprocessed to reduce the dimensionality of the data while retaining the relevant information. Then, we devise an alignment-based similarity measure, based on a rigid registration algorithm (the Iterative Closest Point -ICP). The proposed approach, tested on a real dataset involving 22 proteins, has shown encouraging results in comparison with standard procedures.
\end{abstract}

\section{INTRODUCTION}

In this paper, we address a fundamental issue in structural biology and in structure-based drug design, namely the characterization, for comparative purposes, of a set of macromolecular entities such as proteins (Kahraman and Thornton, 2008). Since structure is more conserved than sequence, the most common approaches rely on the superposition of common structural elements. Such methods allow researchers to compare, with an increasing degree of difficulty, a) different conformations of the same protein, b) homologous proteins and, c) evolutionarily-unrelated proteins.

Traditionally, structural alignment techniques rely on the punctual superposition of correspondent atoms, usually the protein backbones or $\mathrm{C}-\alpha$, in different entries (Shindyalov and Bourne, 1998; Holm and Sander, 1993). A more sophisticated class of protocols considers elements of the protein secondary structures (Jung and Lee, 2000; Chen and Crippen, 2005; Kawabata, 2003). However, these reductionist approaches, based on geometric hashing, do not take into account the physicochemical complexity of the systems. This is because they completely ignore the fields produced by the macromolecule (e.g. the ones experienced by interacting molecules) (Favia, 2011). Furthermore, these methods cannot be safely applied to evolutionarily-distant proteins, due to the lack of sound correspondences between atoms.

To overcome these limits, we herein introduce a new protocol, based on van der Waals potential energies (IUPAC, 1997) calculated at regularly spaced points within a predefined volume. The definition of the volume is case-specific and is usually defined according to the binding site definition. This method allows one a) to compare binding sites and, in a more advanced application, b) to guide a more physicochemically sound structure alignment. Van der Waals interactions between nonbonded atoms can be expressed as a function of their internuclear separation through the Lennard-Jones equations. At each of the regularly spaced points, a virtual atom type is placed and its potential is evaluated. This atom type can be thought of as a chemical probe that experiences the protein fields. The energy at each grid point is determined by the set of parameters supplied for that particular probe, and is estimated as the summation over all atoms of the macromolecule, within a non-bonded cutoff radius, of all pairwise interactions. Different probes experience different fields, according to their assigned chemical features. Taken together, a minimal set of selected probes, namely carbon, oxygen, and hydrogen atoms, can give a useful description of the studied volume based on shape (C probe), $\mathrm{H}$-bond donor, and acceptor propensity ( $\mathrm{H}$ and $\mathrm{O}$ probes, respectively) (see Fig. 1). Once this physicochemical description of the volume is achieved, it can be 

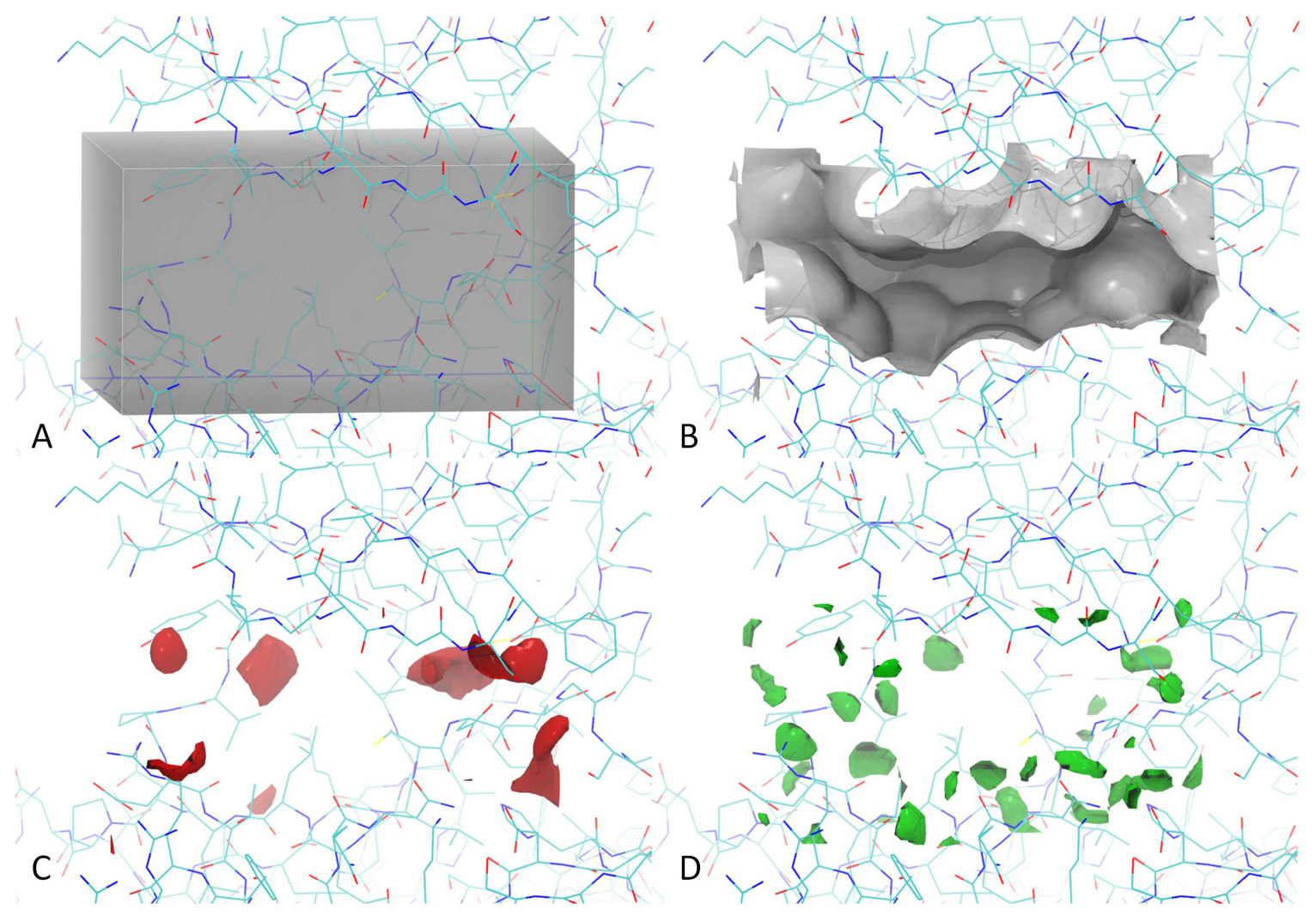

Figure 1: Grid point generation. An enclosing box is defined within a protein active site (A). The grid maps can then be conveniently visualize as isocontour maps. The carbon, oxygen and hydrogen maps are shown in (B), (C) and (D) at $0,-1$ and $-0.6 \mathrm{kcal} / \mathrm{mol}$ isocontour level, respectively. The protein structure is shown in the background of the 4 panels.

used to compare a) different conformations of the same protein and b) different proteins altogether. The presented protocol is similar in spirit to the recently developed protocol for ligand-binding site superposition and comparison based on Atomic Property Fields (Totrov, 2011).

This paper introduces a computational approach able to compare two or more proteins starting from the above-mentioned physicochemical description of their binding site. We call these descriptions atomic grid maps. In particular, the idea is to exploit techniques from the Computer Vision and Pattern Recognition fields to devise similarity between maps in order to understand and highlight relations between different proteins.

The computation of the similarity is carried out in two steps: first, a chemically plausible preprocessing transforms the real-valued potential maps into discrete-valued maps (which we call "meta-maps"); then, the meta-maps are compared using a rigid alignment algorithm (the Iterative Closest Point - ICP ((Besl and McKay, 1992; Chen and Medioni, 1992)), setting the distance between the pair of proteins as the alignment error. This alignment may be performed either by using a single value of the meta-map or by combining together all the values. A similarity clearly expresses the relation between two proteins: a more general view may be obtained by taking a set of proteins, computing all the pairwise distances, and visualizing all the relations through a hierarchical clustering approach (Jain and Dubes, 1988).

The proposed approach has been applied to a real dataset composed of diverse X-ray structures of GSK$3 \beta$, a protein involved in Alzheimer's disease (Hernandez et al., 2009), as extracted from the Worldwide Protein Data Bank (wwPDB) (Berman et al., 2003). The similarity measures obtained with the proposed approach have been compared with those obtained 
through a time-consuming computational procedure based on the comparison of structure-assisted virtual screening ranked distributions (Bottegoni et al., 2011) (which can be considered as the "true" distances). We will show in our experiments that the proposed computational method can approximate these true distances in an encouraging way.

We note that this could be an invaluable drug design tool. This is because representative conformations of a studied protein could be used to run time-demanding molecular simulations (e.g. docking), rather than using the whole ensemble of available structures. More broadly, when applied to unrelated proteins, the methodology could conveniently highlight common hotspots that could be exploited to design multitarget drugs (i.e. molecules capable of binding different proteins). These are particularly useful in treating complex diseases (Morphy and Rankovic, 2006).

The remainder of the paper is organized as follows: Section 2 describes how potentials maps are extracted. In Section 3, we present the proposed approach. Section 4 describes an experimental evaluation that validates the methodology. Finally, in Section 5, conclusions are drawn and future perspectives are envisaged.

\section{POTENTIAL MAPS}

The active sites of the selected structures of GSK$3 \beta$ were first superimposed using the McLachlan algorithm (McLachlan, 1982) as implemented in the program ProFit ${ }^{1}$. Then, van der Waals forces were computed, using the AutoGrid software as distributed with AutoDock4.2 (Morris et al., 2009). AutoGrid solves the Lennard-Jones equations at regularly spaced grid points $(0.375 \AA)$ enclosed in a box centered on the center of mass of the atoms belonging to the active site, spanning $14.25,11.25$ and $21 \AA$ along the three axes. To give an accurate description of the site, three diverse atom probes were placed at each node of the grid to sense the protein environment, namely the carbon, hydrogen and oxygen probes. In particular, the carbon probe accounts for the shape and hydrophobic features of the binding site, while the hydrogen and the oxygen probes account for the hydrogen bond propensity. Pairwise atomic interactions are approximated through the following equation:

$$
V(r)=\frac{C_{n}}{r^{n}}-\frac{C_{m}}{r^{m}}=C_{n} r^{-n}-C_{m} r^{-m}
$$

\footnotetext{
${ }^{1}$ A.C.R. Martin http://www.bioinf.org.uk/software/profit/
}

Here, $m$ and $n$ are integers, $C_{n}$ and $C_{m}$ are constants whose values vary according to the type of atoms and probes involved and $r$ is the distance between them. At distances shorter than the equilibrium distance (in correspondence to the minimum of the function), the potential energy function increases rapidly (i.e. the probe clashes with the protein), while at long distances the function tends to zero (i.e. the probe does not feel the presence of the protein).

\section{THE PROPOSED APPROACH}

The goal of the proposed approach is to characterize a set of proteins, each one described by a set of potentials maps. The idea is to highlight the relations between the different proteins of the chosen set by devising a similarity measure, which could potentially be used in a clustering scenario to highlight all the possible relations. Some different distances are defined and described in Sect. 3.2. These are all based on a chemically sound preprocessing algorithm used to simplify the potential maps, as described in the next Section.

\subsection{Preprocessing of data: the meta-maps}

AutoGrid produces real-valued potential maps. As such, they are difficult to interpret. Hence, we parsed the three Auto-Grid readouts to yield a single map that retains all the relevant information. A few considerations must be made here:

- the oxygen and hydrogen probes are mutually exclusive;

- both are considered to be more relevant, from a chemical perspective, than the carbon probe;

- we were only interested in negative values (i.e. when probes and protein do not clash)

- after a heuristically defined cutoff, small differences in potential energies are negligible.

Bearing this in mind, every position of the potential maps may be described with one of four different values:

- value '1': if the oxygen probe, in this position, recorded a potential energy lower than -1 $\mathrm{kcal} / \mathrm{mol}$;

- value '-1': if the hydrogen probe, in this position, recorded a value lower than $-0.6 \mathrm{kcal} / \mathrm{mol}$;

- value '0': if the carbon probe, in this position, recorded any negative value of the potential energy function; 
- no value if none of the above criteria were fulfilled in this position;

In doing so, a net compression of the data is possible, yet the relevant information is retained and conveniently encoded into a single, ternary grid map.

\subsection{Devising the similarity}

Once the meta-maps have been obtained, the next step is to define the similarity measure. One reasonable strategy is to link the similarity to the alignment of the meta-maps, in order to measure, in some sense, how dissimilar two maps remain after maximizing the overlap between them (it may also be seen as the opposite of the overlap ratio between two maps).

In particular, we investigated two different approaches:

1. Alignment based on a single value. Here, we selected only those meta-maps points with a specific value $(-1,0$ or 1$)$. In this way, the alignment was transformed into a simpler problem of registration of point clouds - where the term "registration" describes the geometric alignment of a pair of 3D data-point sets. This is a well-known problem in computer vision (Trucco and Verri, 1998), and many techniques to solve it have been proposed in the past. One of the most famous is the Iterative Closest Point (ICP - (Besl and McKay, 1992; Chen and Medioni, 1992)), briefly summarized later in this section;

2. Alignment based on all values: the distance was computed by simultaneously using all the values of the metamaps.

Before entering into the details, we will review the Iterative Closest Point (ICP) algorithm (Besl and McKay, 1992; Chen and Medioni, 1992).

\subsubsection{Iterative Closest Point algorithm}

Let us suppose that we have two sets of 3D points, $V^{i}$ and $V^{j}$. The registration consists of finding a 3D transformation which, when applied to $V^{j}$, minimizes the distance between the two point sets. In general, point correspondences are unknown. For each point $y_{i}$ from the set $V^{j}$, there exists at least one point on the surface of $V^{i}$ that is closer to $y_{i}$ than all the other points in $V^{i}$. This is the closest point, $x_{i}$. The basic idea behind the ICP algorithm is that, under certain conditions, closest points are a reasonable approximation to the true point correspondences. The ICP algorithm can be summarized as follows:

1. For each point in $V^{j}$, compute the closest point in $V^{i}$
2. With the correspondence from step 1 , compute the incremental transformation $\left(R^{i, j}, t^{i, j}\right)$;

3. Apply the incremental transformation from step 2 to the set $V^{j}$;

4. If the change in total mean square error is less than a threshold, terminate. Otherwise, go to step 1.

Besl and McKay (Besl and McKay, 1992) proved that this algorithm is guaranteed to converge monotonically to a local minimum of the mean square error. Thus, a good initialization is required. To overcome this problem, we manually pre-aligned the proteins in our experiments. For step 2, efficient, noniterative solutions to this problem (known as the point set registration problem) were compared in (Lorusso et al., 1997). The solution based on singular value decomposition was found to be the best in terms of accuracy and stability.

After the convergence of the algorithm, the total mean square error represents the registration error between the two sets of points.

After the convergence of the algorithm, the total mean square error represents the registration error between the two sets of points.

\subsubsection{Single value analysis}

Given two proteins to be compared, the distance is computed via the following steps:

1. for every protein, the three potential maps are preprocessed to produce the corresponding metamap;

2. from the meta-map, only points with a specific value are extracted (for example, all points with '0' value). This results in a cloud of 3D points;

3. the two 3D point clouds (relative to the two proteins) are registered through the ICP algorithm. The registration error represents the final distance.

\subsubsection{Multiple values analysis}

The previous approach is of course limited by the fact that the meta-maps are decomposed in three different non-overlapping sets, which are used alone - in this sense using only partial information. It seems reasonable, therefore, to try to develop a method that can integrate and use all the information present in the meta-maps. From a very general Pattern Recognition point of view, this problem may be contextualized in the Multiclassifier theory (also called Multimodal or Fusion theory, depending on the context). These theories aim to integrate the potentially complementary information provided by different methodologies/representations in a particular problem, by ex- 
ploiting the different peculiarities of the fused techniques. This theory, first introduced in the classification context (Ho et al., 1994; Kittler et al., 1998; Melnik et al., 2004) and, more recently, in the clustering context ((Topchy et al., 2005; Fred and Jain, 2005) and references therein), seems to be particularly suited for the context we are investigating. In particular, the information fusion could be performed at three different levels (Ross and Jain, 2004): data or feature level, where feature representations are combined; score level, where scores derived from different modalities (e.g. similarities) are composed to get a new score; and decision level, where the final outputs (i.e. clusterings or trees) of multiple strategies are consolidated.

In this preliminary analysis, we investigate a very simple yet promising approach, aimed at performing an integration at the distance level. The idea is to derive three different single-value-based registrations, leading to three different distance measures, which are finally integrated in a final distance measure. In more detail, given a protein pair $(i, j)$, the starting point is represented by the three distances $d_{-1}(i, j), d_{0}(i, j)$ and $d_{+1}(i, j)$. The main goal is to combine them in order to obtain a more meaningful one. Clearly, in the multiclassifier taxonomy provided above, we are performing score-level fusion. In general, fusion at score level is preferred (Duin and Tax, 2000; Tax et al., 2000). This is because it is relatively easy to access and combine scores produced by the different modalities. Furthermore, some studies have reported its superiority against feature-level fusion and decision-level fusion - e.g. (Kumar et al., 2003). Many techniques have been proposed in the past, with different characteristics. Here we use two rules:

1. Mean rule: in this case the three distances are simply averaged.

$$
d_{M}(i, j)=\frac{d_{-1}(i, j)+d_{0}(i, j)+d_{+1}(i, j)}{3}
$$

Despite its simplicity, this rule (also called SUM rule) has proven to be very competitive in many applications, while maintaining many interesting theoretical properties (Kittler et al., 1998).

2. Weighted mean rule: in this case, the new distance is a convex combination of the three distances:

$d_{W M}(i, j)=\alpha_{-1} d_{-1}(i, j)+\alpha_{0} d_{0}(i, j)+\alpha_{+1} d_{+1}(i, j)$

such that

$$
\alpha_{-1}+\alpha_{0}+\alpha_{+1}=1
$$

Defining the three weights may be difficult. An interesting theoretical analysis of linear combin- ers for multiple classifiers systems can be found in (Fumera and Roli, 2005). Here, we performed a large scale analysis, trying many different values, and selecting a posteriori the best triplet. We are aware that this a posteriori choice is not optimal, and we are currently experimenting with an alternative and cleverer search strategy, which is based on problem-driven information (following the rationale applied in the phylogeny context by (Bicego et al., 2007)).

\section{EXPERIMENTAL RESULTS}

The proposed approach was validated using a protein dataset comprising 22 proteins. In particular, the different distances were computed following the procedure described in the previous section. In the specific case of comparing protein structures, one procedure to determine a reliable distance between them can be achieved through an undeniably timeconsuming computational procedure (see below for details). The main goal of this experimental evaluation was to compare, within the specific set of proteins, these "true similarities" (similarities obtained via docking) with the similarity obtained by our approach. We carried out both a qualitative and a quantitative analysis. For the qualitative analyses, we used the proposed distances to derive a dendrogram - via a standard agglomerative hierarchical clustering approach (Jain and Dubes, 1988). We made some observations by comparing the trees obtained with our distances and those obtained with the true ones. For the quantitative analyses, the proposed similarities were compared with the true ones using the Mantel test (Mantel, 1967).

\subsection{The dataset}

The protein dataset was composed of $22 \mathrm{X}$-ray protein structures of GSK-3 $\beta$ as available at the wwPDB. Being obtained under different experimental conditions, the structures were available at different crystallographic resolutions and were solved either alone or in complex with structurally diverse inhibitors (see Tab. 1). As a direct consequence of this, the selected PDB entries were conformationally distinct from each other and each represented an experimentally observed moment of the protein dynamics (see Fig. 2).

GSK-3 $\beta$ is a pharmacological target for Alzheimer's disease and, due to the abundance of experimentally determined data, structure-assisted methods, such as molecular docking, are widely used 


\begin{tabular}{|c|c|c|c|}
\hline $\begin{array}{l}\text { PDB } \\
\text { Entry } \\
\text { (Ref) }\end{array}$ & $\begin{array}{l}\text { Resolution } \\
\text { (Å) }\end{array}$ & Chain:Residues & Inhibitor \\
\hline $1 \mathrm{GNG}$ & 2.60 & $\begin{array}{l}\text { A: } 36-385 \\
B: 28-384\end{array}$ & -- \\
\hline $1 \mathrm{H} 8 \mathrm{~F}$ & 2.80 & $\begin{array}{l}\text { A: } 35-386 \\
\text { B: } 35-384\end{array}$ & -- \\
\hline 1109 & 2.70 & $\begin{array}{c}\text { A: } 25-384 \text { (missing 120-126, 286-300) } \\
\text { B: } 37-382 \text { (missing 286-290) }\end{array}$ & \\
\hline 1J1B & 1.80 & $\begin{array}{l}\text { A:35-388 } \\
\text { B: } 23-386\end{array}$ & $\begin{array}{l}\text { (ANP) phosphoaminophosphonic acid-adenilate } \\
\text { ester }\end{array}$ \\
\hline $1 \mathrm{J1C}$ & 2.10 & $\begin{array}{l}\text { A:35-388 } \\
\text { B:23-386 }\end{array}$ & (ADP) adenosine- $5^{\prime}$-diphosphate \\
\hline $1 P Y X$ & 2.40 & $\begin{array}{c}\text { A:35-386 (missing 120-124, 287-290) } \\
\text { B:35-386 (missing 120-124, 285-290, 384- } \\
386 \text { ) }\end{array}$ & $\begin{array}{l}\text { (ANP) phosphoaminophosphonic acid-adenilate } \\
\text { ester }\end{array}$ \\
\hline 1Q3D & 2.20 & $\begin{array}{c}\text { A:35-385 (missing 120-124,287-292) } \\
\text { B:35-385 (missing 120-123,287- } \\
292,384,385 \text { ) }\end{array}$ & (STU) staurosporine \\
\hline 1Q3W & 2.30 & $\begin{array}{c}\text { A:35-385 (missing } 121-124,288-291) \\
\text { B:35-385 (missing } 121-123,287-291,384- \\
385)\end{array}$ & (ATU) Alsterpaullone \\
\hline $1 Q 41$ & 2.10 & $\begin{array}{l}\text { A:35-386 (missing 120-125,285-291) } \\
\text { B:35-386 (missing 120-125,384-386) }\end{array}$ & (IXM) Indirubin-3'-monoxime \\
\hline $1 \mathrm{Q} 4 \mathrm{~L}$ & 2.77 & $\begin{array}{l}\text { A:35-386 (missing 121-123,286-292) } \\
\text { B:35-386 (missing 292-299,384-386) }\end{array}$ & (679) I-5 \\
\hline $1 Q 5 K$ & 1.94 & $\begin{array}{l}\text { A:35-384 (missing 120,121,287-289) } \\
\text { B:35-386 (missing 287-289,295-297) }\end{array}$ & (TMU) AR-A014418 \\
\hline $1 \mathrm{ROE}$ & 2.25 & A/B:35-383 (missing 120-124) & $\begin{array}{l}\text { (DFN) 3-(3-\{[(2S)-2,3- } \\
\text { dihydroxypropyl]amino\}phenyl)-4-(5-fluoro-1- } \\
\text { methyl-1H-indol-3-yl)-1H-pyrrole-2,5-dione }\end{array}$ \\
\hline 1UV5 & 2.80 & A:35-383 & (BRW) 6-bromoindirubin-3'-oxime \\
\hline 2JLD & 2.35 & $\begin{array}{c}\text { A:35-385 (missing 120,290) } \\
\text { B:35-384 (missing 292) }\end{array}$ & (AG1) ruthenium pyridocarbazole \\
\hline $205 \mathrm{~K}$ & 3.20 & $A: 35-384$ & (HBM) 7-hydroxy-1H-benzoimidazole \\
\hline 20W3 & 2.80 & $\begin{array}{c}\text { A:35-386 (missing 119-122, 386) } \\
\text { B:35-386 }\end{array}$ & (BIM) Bis(indoyl)maleimide-para-pyridinophane \\
\hline 3DU8 & 2.20 & $\begin{array}{l}\text { A:35-382 (missing 120-125,287-292) } \\
\text { B: 35-385 (missing 120-125,287-292) }\end{array}$ & $\begin{array}{l}\text { (553)(7S)-2-(2-aminopyrimidin-4-yl)-7-(2- } \\
\text { fluoroethyl)-1,5,6,7-tetrahydropyrrolo[3,2- } \\
\text { c]pyridin-4-one }\end{array}$ \\
\hline $3 F 7 Z$ & 2.40 & $\begin{array}{l}\text { A:35-383 (missing 122-124,288-294) } \\
B: 35-383 \text { (missing 120-125,290-293) }\end{array}$ & $\begin{array}{l}\text { (340) 2-(1,3-benzodioxol-5-yl)-5-[(3-fluoro-4- } \\
\text { methoxy-phenyl)methylsulfanyl]-1,3,4-oxadiazole }\end{array}$ \\
\hline \multirow{2}{*}{$3 F 88$} & \multirow{2}{*}{2.60} & \multirow[b]{2}{*}{ A/B:35-383 (missing 120-125,288,289) } & (2HT) 3-methylbenzonitrile \\
\hline & & & $\begin{array}{l}\text { (3HT) 5-[3-(4-methoxyphenyl)benzimidazol-5-yl]- } \\
3 \mathrm{H}-1,3,4-\text { oxadiazole-2-thione }\end{array}$ \\
\hline $314 \mathrm{~B}$ & 2.30 & $\begin{array}{l}\text { A:33-385 } \\
\text { B:36-382 }\end{array}$ & $\begin{array}{l}\text { (Z48) N-[(1S)-2-hydroxy-1-phenylethyl]-4-[5- } \\
\text { methyl- 2-(phenylamino)pyrimidin-4-yl]-1H- } \\
\text { pyrrole- 2-carboxamide }\end{array}$ \\
\hline $3 G B 2$ & 2.40 & A:35-119;125-286; 290-383 & $\begin{array}{l}\text { (G3B) 2-methyl-5-(3-\{4-[(S)-methylsulfinyl]phenyl\}- } \\
\text { 1-benzofuran-5-yl)-1,3,4-oxadiazole }\end{array}$ \\
\hline 3L1S & 2.90 & $\begin{array}{c}\text { A:25-118 (missing } 32,33,34) \\
126-285 ; 300-384 \\
\text { B:36-422 (missing } 120-121 ; 286-300 ; 383- \\
420 \text { ) }\end{array}$ & $\begin{array}{l}\text { (Z92) (4E)-4-[(4-chlorophenyl)hydrazono]-5-(3,4- } \\
\text { dimethoxyphenyl)-2,4-dihydro-3H-pyrazol-3-one }\end{array}$ \\
\hline
\end{tabular}

Table 1: Detailed list of the structures included in the dataset used in this study.

in drug discovery pipelines, both in academia and in industry.

Before starting a virtual ligand screening, when a number of X-ray structures of the same protein are available, one fundamental question that should be addressed is: how diverse are the included structures?
Having access to this information would allow researchers to select only a minimal subset of entries, which preserves most of the relevant variance, for running time-demanding docking simulations. Unfortunately, it is still not possible to assess, a priori, redundancy between entries for virtual screening pur- 


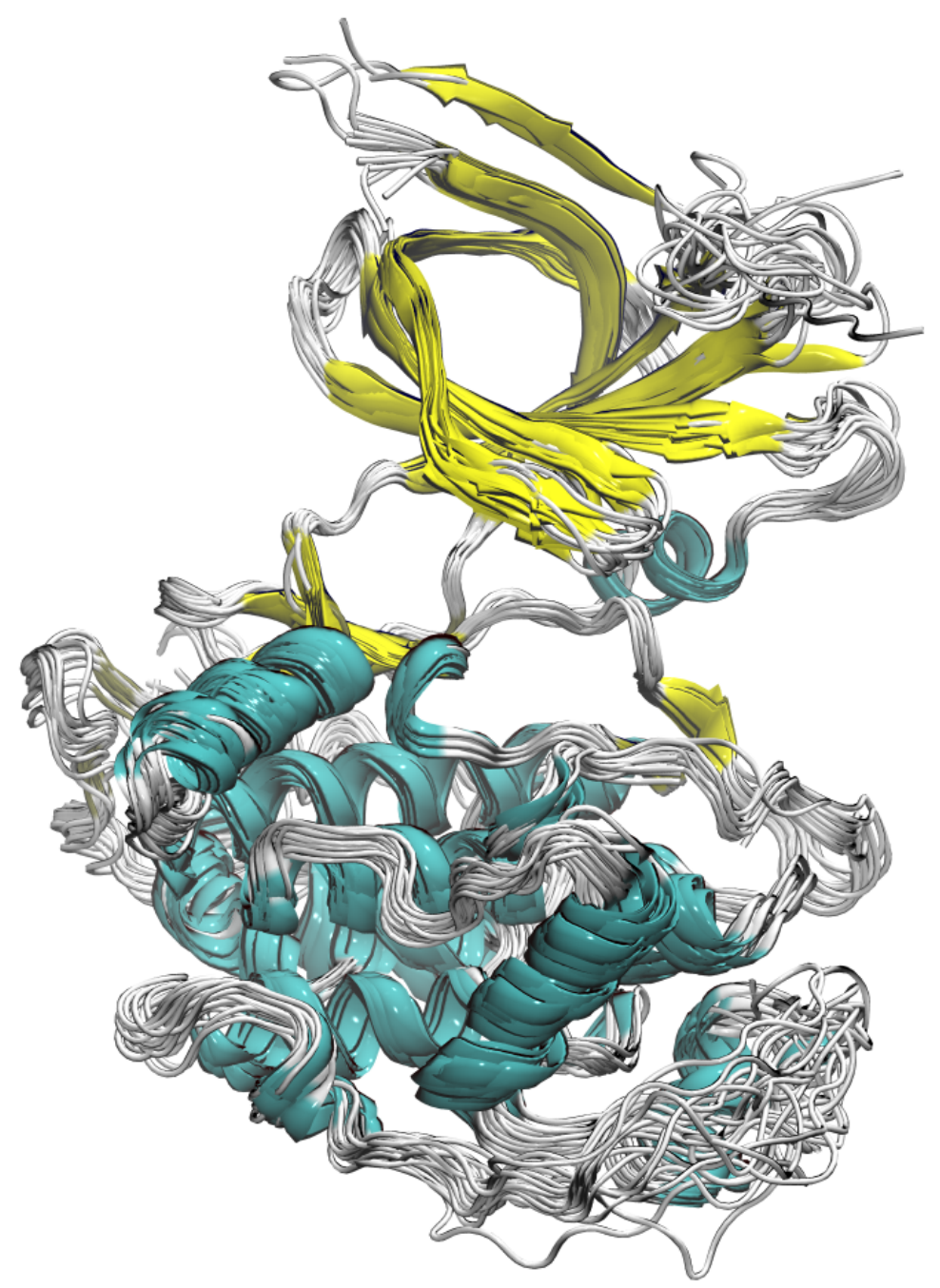

Figure 2: Structural superposition of the $22 \mathrm{PDB}$ entries employed in this study. The structures are depicted in ribbon and coloured according to the secondary structure elements.

poses.

To this end, the set used in this study was first characterized using a standard molecular docking simulation, described below. This procedure is computationally demanding, and may be unfeasible in real life scenarios for larger datasets of protein structures. Nevertheless, it allowed us to generate a ground truth, to be used retrospectively to assess the accuracy of the proposed method.

\subsubsection{The ground truth}

In a molecular docking simulation, a molecule is computationally docked at a protein active site with the aim of predicting possible modes of interaction between the two. A number of poses are generated and ranked according to the associated estimation of the binding energy (score). Top scoring poses are usually the ones considered, since these are supposedly the ones that occur experimentally. In a virtual screening effort, a number of molecules are docked at an enzyme-binding site and their bind- 
ing affinities are estimated. Assuming that, for each molecule, only one pose is taken into account (i.e. the top scoring one), the final ranked distribution will reflect the molecular preferences of a given protein. In fact, different proteins will reward different classes of molecules, characterized by specific physico-chemical features. An extension of this consideration is that different conformations of the same protein will reward different molecules too. This is because docking algorithms treat these conformations as if they were different molecular entities altogether. In this context, the ranked distribution indirectly describes the physicochemical characteristics of a protein. We can think of these as fingerprints, where each position in the rank represents a bit and the unique molecule in that position represents its assigned value. To obtain meaningful results, a reasonable number of molecules should be used. The set should be big enough to allow a fine distinction between proteins, yet small enough to be computationally feasible. It should be composed of chemically diverse entries in order to eliminate noise-generating redundancy. In this procedure, a set of 6354 diverse compounds was docked at each of the 22 protein structures. Pearsons correlation coefficients calculated between the obtained ranks represent the final distance, on which the clustering procedure can be built.

In a real life scenario, where millions of compounds and several tens of proteins can be involved, using ranked distributions for clustering purposes could be unfeasible. Grid-driven clustering offers a quicker approach to this challenge. Moreover, mapsdriven analysis can be more easily interpreted from a chemical standpoint.

\subsection{Quantitative evaluation}

Table 2 reports the correlation coefficients between the proposed methods and the ground truth distances (we note that the correlation, in this case, takes values from -1 to $+1-$ the higher the value, the better the result). In addition to the correlation factor, a p-value is also provided, which measures the probability that the same correlation is obtained if randomly permuting the rows or the columns of one of the matrices. Some observations may be drawn from the table:

- the information carried out by the three singlevalue maps is rather different: the analysis based on the single values 0 and +1 is not as good as the analysis made with the value -1 - even if it shows a positive correlation with the ground truth

- this is confirmed by the results obtained with the mean rule (which gives the same weight to all the maps); a proper weighing of the three distances yields better results.

- combining the three distances improves the single-value analysis, thus confirming the complementary information present in the original sources

\subsection{Qualitative evaluation}

Given the distance, a qualitative analysis could be carried out by looking at trees obtained via the application of clustering techniques to the similarity matrices - the proposed ones and the ground truth ones. In particular, dendrograms were obtained with the UPGMA clustering algorithm (as implemented in the Phylip Package $^{2}$ ). In Fig. 3 two trees are reported, namely the ground truth one and the one obtained with the -1 map.

Rather than looking at the global similarity between trees, a perceptive comparison should focus mostly on the local matches. This is because, above a defined cutoff, distances between entries tend to be less consistent. Indeed, the map-driven tree reproduced nicely some of the trends recorded by the docking ranks. For instance, entries $1 \mathrm{GNG}, 1 \mathrm{I} 09,2 \mathrm{O} 5 \mathrm{~K}$ and $1 \mathrm{H} 8 \mathrm{~F}$ were singletons, according to the ground truth. As illustrated in Fig. 3, the oxygen mapdriven tree put 5 entries distant from the rest. Four of those entries were indeed the ground truth singletons, while the remaining entry (i.e. 1QW3) was erroneously recognized as close to $1 \mathrm{H} 8 \mathrm{~F}$. From a biological perspective, 3 out of those 4 entries were peculiar cases, being the only proteins of the set whose crystals lacked a molecule bound. Another very interesting achievement is the pairing of $1 \mathrm{~J} 1 \mathrm{~B}$ and $1 \mathrm{~J} 1 \mathrm{C}$. Those Xray structures in fact showed very similar molecules bound, which in turn yielded highly comparable conformational rearrangements. Another notable result was found for the cluster composed of entries 3L1S, 1UV5, 1Q41, 1Q5K and 314B, which perfectly matched the one found in the ground truth. Entries 3F88, 3DU8 and 1PYX clustered together in the map-driven tree. The same trend was found in the ground truth, with the exception of entry 3F7Z, which was missing in the former. Overall, the remarkable resemblance between the ground truth and the mapdriven trees speaks to the accuracy of the proposed methodology in finding hidden relevant chemical patterns in protein structures. Nonetheless, there is room for improvement. For instance, in a less reductionist approach, more atom probes could be used.

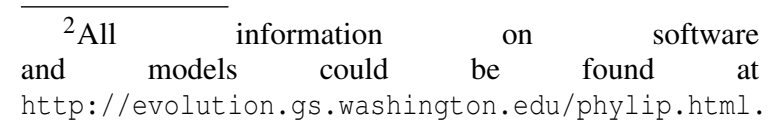




\begin{tabular}{|l|c|c|}
\hline Method & Correlation & p-value \\
\hline Single value (-1) & 0.708 & 0.001 \\
Single value (0) & 0.240 & 0.071 \\
Single value (+1) & 0.305 & 0.020 \\
Multi values - mean rule & 0.548 & 0.001 \\
Multi values - weighted mean rule & 0.721 & 0.001 \\
\hline
\end{tabular}

Table 2: Correlations computed with the Mantel test for the different approaches.

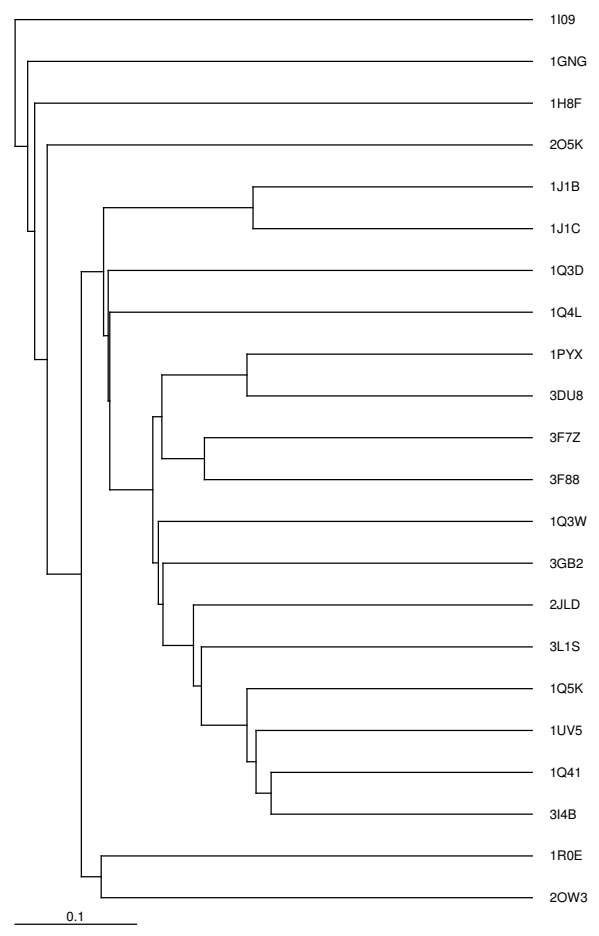

(a)

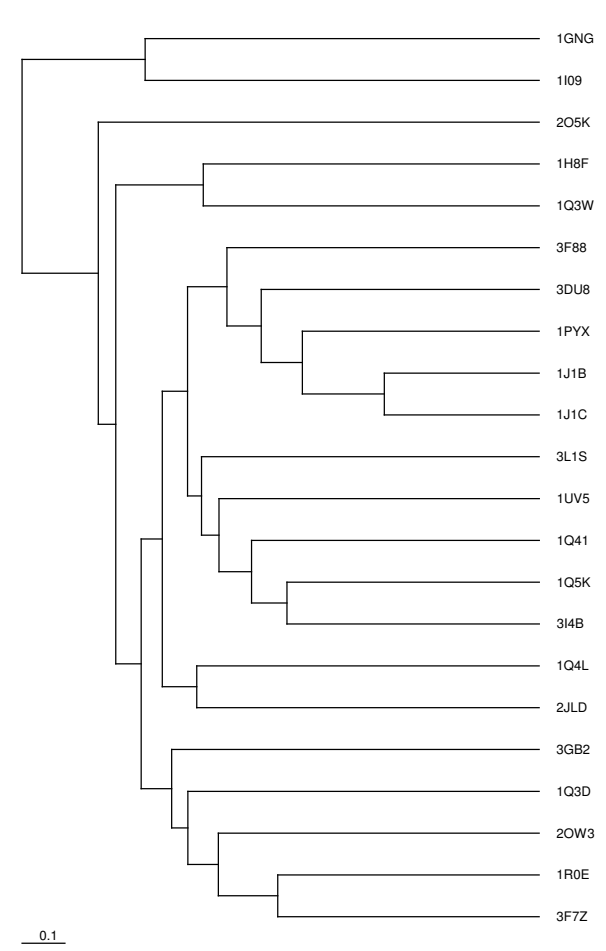

(b)

Figure 3: Trees obtained with UPGMA of the phylip package: (a) ground truth; (b) obtained with '-1' map

\section{CONCLUSIONS}

In this paper, we proposed a novel computational approach to comparing two or more proteins, starting from a physico-chemical description of their binding site (atomic grid maps). These maps were preprocessed via a chemically plausible procedure that simplified the data while retaining the relevant information. Different alignment-based similarity measures were proposed based on a rigid registration algorithm. The proposed approach was tested on a real dataset involving 22 proteins. Retrospective evaluations, both qualitative and quantitative, proved the feasibility of the method.

\section{ACKNOWLEDGEMENTS}

We kindly acknowledge the IIT computational platform initiative for providing computer time. We thank Grace Fox for editing and proofreading the manuscript. 


\section{REFERENCES}

Berman, H., Henrick, K., and Nakamura, H. (2003). Announcing the worldwide protein data bank. Nat Struct Biol, 10:980.

Besl, P. and McKay, N. (1992). A method for registration of 3d shapes. IEEE Trans. on Pattern Analysis and Machine Intelligence, 14:239-256.

Bicego, M., Dellaglio, F., and Felis, G. (2007). Multimodal phylogeny for taxonomy: Integrating information from nucleotide and amino acid sequences. J. Bioinformatics and Computational Biology, 5(5):10691085.

Bottegoni, G., Rocchia, W., Rueda, M., Abagyan, R., and Cavalli, A. (2011). Systematic exploitation of multiple receptor conformations for virtual ligand screening. Plos One. in press.

Chen, Y. and Crippen, G. (2005). A novel approach to structural alignment using realistic structural and environmental information. Protein Sci, 14:2935-2946.

Chen, Y. and Medioni, G. (1992). Object modeling by registration of multiple range images. Image Vision Computing, 10:145155.

Duin, R. and Tax, D. (2000). Experiments with classifier combining rules. In Proc. Workshop on Multiple Classifier Systems, pages 16-29.

Favia, A. (2011). Theoretical and computational approaches to ligand-based drug discovery. Frontiers in Bioscience, 16:1276-1290.

Fred, A. and Jain, A. (2005). Combining multiple clusterings using evidence accumulation. IEEE Trans. on Pattern Analysis and Machine Intelligence, 27(6):835-850.

Fumera, G. and Roli, F. (2005). A theoretical and experimental analysis of linear combiners for multiple classifier systems. IEEE Trans. on Pattern Analysis and Machine Intelligence, 27(6):942-956.

Hernandez, F., De Barreda, E., Fuster-Matanzo, A., GoniOliver, P., Lucas, J., and Avila, J. (2009). The role of gsk3 in alzheimer disease. Brain Res Bull, 80:248250.

Ho, T., Hull, J., and Stihari, S. (1994). Decision combination in multiple classifier systems. IEEE Trans. on Pattern Analysis and Machine Intelligence, 16(1):6675.

Holm, L. and Sander, C. (1993). Protein structure comparison by alignment of distance matrices. $\mathrm{J} \mathrm{Mol} \mathrm{Biol,}$ 233:123-138.

IUPAC (1997). Compendium of chemical terminology. (the "Gold Book"). Online corrected version: (1994) "Van der Waals forces".

Jain, A. and Dubes, R. (1988). Algorithms for clustering data. Prentice Hall.

Jung, J. and Lee, B. (2000). Protein structure alignment using environmental profiles. Protein Eng, 13:535543.

Kahraman, A. and Thornton, J. (2008). Methods to characterize the structure of enzyme binding sites. In Schwede, T. and Peitsch, M., editors, Computational
Structural Biology - Methods and Applications, volume 1, pages 189-221. World Scientific Publishing Co.

Kawabata, T. (2003). Matras: A program for protein 3d structure comparison. Nucleic Acids Res, 31:33673369.

Kittler, J., Hatef, M., Duin, R., and Matas, J. (1998). On combining classifiers. IEEE Trans. on Pattern Analysis and Machine Intelligence, 20(3):226-239.

Kumar, A., Wong, D., Shen, H., and Flynn, P. (2003). Personal verification using palmprint and hand geometry biometric. In Proc. of Int. Conf. on Audio and Video-based biometric person authentication, pages 668-678.

Lorusso, A., Eggert, D., and Fisher, R. (1997). A comparison of four algorithms for estimating 3-d rigid transformations. Machine Vision Applications, 9:272290.

Mantel, N. (1967). The detection of disease clustering and a generalized regression approach. Cancer Research, 27(2):209-220.

McLachlan, A. (1982). Rapid comparison of protein structures. Acta Cryst, A38:871-873.

Melnik, O., Vardi, Y., and Zhang, C.-H. (2004). Mixed group ranks: Preference and confidence in classifier combination. IEEE Trans. on Pattern Analysis and Machine Intelligence, 26(8):973-981.

Morphy, R. and Rankovic, Z. (2006). The physicochemical challenges of designing multiple ligands. J Med Chem, 49:4961-4970.

Morris, G., Huey, R., Lindstrom, W., Sanner, M., Belew, R., Goodsell, D., and Olson, A. (2009). Autodock4 and autodocktools4: Automated docking with selective receptor flexibility. J Comput Chem, 30:2785-2791.

Ross, A. and Jain, A. (2004). Multimodal biometrics: an overview. In Proc. of European Signal Processing Conference, pages 1221-1224.

Shindyalov, I. and Bourne, P. (1998). Protein structure alignment by incremental combinatorial extension (ce) of the optimal path. Protein Eng., 11:739_ 747.

Tax, D., Breukelen, M., Duin, R., and Kittler, J. (2000). Combining classifiers by averaging or by multiplying? Pattern Recognition, 33:1475-1485.

Topchy, A., Jain, A., and Punch, W. (2005). Clustering ensembles: models of consensus and weak partitions. IEEE Trans. on Pattern Analysis and Machine Intelligence, 27(12):1866-1881.

Totrov, M. (2011). Ligand binding site superposition and comparison based on atomic property fields: identification of distant homologues, convergent evolution and pdb-wide clustering of binding sites. BMC Bioinformatics, 12(Suppl 1):S35.

Trucco, E. and Verri, A. (1998). Introductory Techniques for 3-D Computer Vision. Prentice-Hall. 